three-dimensional in which only two-dimensional curved bodies can exist without loosing the continuity, while the three-dimensional Riemannian manifold cannot be realized without being developed and slashed into flat pieces accompanied by dislocations.

\section{\&7. Conclusion}

We have obtained the fundamental equations (6.1) of yielding by means of a series of purely logical analy'ses. The equation of equilibrium is shown to coincide entirely with what has been anticipated by the geometrical consideration in a previous paper ${ }^{1)}$, as can be expected. The theory of yielding is thus formulated as a typical problem. concerning the boundary conditions and the chatac teristic values. We are now in a position to demonsta cate the validity of ou proposal by analyzing specifiexamples.

\section{Bibliography}

1) K. Kondo: A Proposal of a New Theory concerning the Yielding of Materials based on Riemannian Geometry I and II. Journ. J̦apan Soc. Appl. Mech., Vol. 2, No.11(1949) pp. $123 / 128$. No. 12 , pp $146 / 151$.

2) K. KONDO: On the Dislocation, the Group of Holonomy and the Theory of Yielding. ibid. Vol. 3. No. 17 (1950) pp. 107/110.

\title{
The Mathematical Analyses of the Yield Point. I* Uniform Stress
}

\author{
By Kazuo Kondo, University of Tokyo
}

\section{Introduction}

We have pointed out that the clear yield point of such a material as mild steel should be mathe; matically nothing else than the critical point where the test-piece lodses its Euclidean metric as a threedimensional continous manifold. 1) If the following three important points lying at the basis of the proposed hypothesis can be regarded sound enough the fundamental equations which have been propounded in the previous papers $\left.{ }^{12}{ }_{3}^{2}\right)_{3} 3$, the analytical expressions, namely the equation of equilibrium and the boundary conditions, may be satifjed.

The principal parts of the assumptions are

$1^{\circ}$ That in each element of the material which has been subjected only to continuous compatible deformation dislocations appear at the yield point where they get free from the restraint of the condition of compatibility in the three-dimensional Euclidean space;

$2^{\circ}$ That a certain resistance takes place against the deformation accompanied by dislocations, the point of balance between such a resistance and the motive force to produce the dislocation being regarded as the yield point. The yielding is, therefore, a sort of critical phenomena concerning statical stability and instability ;

$3^{\circ}$ That there is a limit of the linear dimension under which such mean "properties of materials as the conception of elasticity cannot hold and accordingly the usual theory of elasticity must be modified.

The whole system of the theory is logically deduced from the above combined with the fundamental notions of mechanics and thermodynamics, without artificial modifications or unreasonable? simplifications of the actual phenomena. The conceptions of Riemannian Geometry have necessarily. been introduced as languages which are the most

\footnotetext{
* Read at the 16th Session of the Japan. Society for Applied Mechanics, July 24th 1949.

Received on Dac. 19.1949.
} 
suitable to express such entities mathematically. By a more critical observations the reader may recognize that $3^{\circ}$ is not independent of $1^{\circ}$ and $2^{\circ}$ And if $3^{\circ}$ is considered rather too probable because of the microscopic structure of the material ${ }^{1)}$, we may regard ourselves well grounded. The appearances of such abstract expressions are however likely to give an impression as if they were quite independent of the real entities that they have to represent. It may inv6ke some doubts concerning the soundness of our point of view even if no error is found in the course of reasoning. Or all the arguments may appear as if they were illusive even if the starting point is permitted. In order to get rid of such encumbering doubts, it is necessary to perform analyses of the representative examples of the yielding phenomena so that we may obtain the actual values of yield points for comparison. Therefore we shall start now on a series of mathematical analyses upon the problems of characteristic values with respect to the condition of yielding for the standard cases conceivable.

This fitst report treats of the yielding by the uniform stress which is the simplest and for which we have already a great many of experimental researches and criticisms of the existing hypothesis proposed up to this time. Some fundamental principles proper to the problems of this sort should be considered at the same time. They should be related to the essential natures of the phenomena of yielding about which we have not yet had the occasion to discuss in detail.

Certainly there must be found an important role for the modern theory of matter concerning the interpretation ot this sort of phenomena. But conceptions of such a specific branch of science are not required for our present purposes. Whatever may be the microscopic structure, its contributions to the yielding phenomena must be so formulated as shown here.

\section{\$1. The General Principle}

The fundamental criterions in the theory of yield- ing have been expressed by the field equation of equilibrium

$$
B \Delta \Delta w+\sigma^{i j} \frac{\partial^{2} w}{\partial x^{i} \partial x^{j}}=0,
$$

and the conditions for the free bourdary:

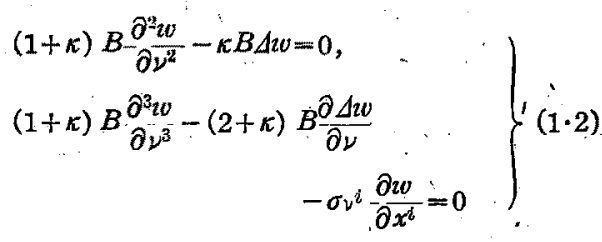

where each symbol has the same meaning as in the previous papers ${ }^{1}, 3$; namely $B$ and $\kappa$ are the material constants, $\sigma^{i j}$ and $\sigma \nu^{i}$ the components of the stress ternsor, the coordinates $x^{i},(i=1,2,3)$ of a rectangular cartesion system and $\nu$ the surface normal. The function $w$ represents the dislocation function. 2),3)

Since the structure of the whole theory is a three-dimensional analogue of the theory of buckling of a flat plate as a problem of characteristic value, the functional form of $w$ must also be analogous and its distribution can vary in accordance with the shape and size of the test-piece as well as with the condition of load. All the experimental evidences suggest in confirmation of such tendency, although the influences do not appear to be so remarkable as in the case of the buckling of a plate. In consideration of such a circumstance, we can introduce a simplification of the problem of characteristic values consistent with the practical conditions.

It appears that the existence of the boundary at the contrary side does not give any essential influence on the condition of "yielding, as can be obserbed in the case of tension test with respect to the sizes and certain kinds of differences of the shapes of the test-pieces. It signifies localization of the play-field of the disturbance. As is well known, the effect of such localization is not so remarkable in lower-dimensional cases. It appears the less the less the number of dimensions becomes, namely none for the case of the columa, a 
little for the flat plate, more remarkable for curved shells. -

Hence the localized disturbances must naturally be far more predominant in the case of threedimensional manifold, of which the yielding has the same type of mathematical expressions as that of the buckling. Based on the consideration of this kind, we shall introduce the following auxiliary criterions, which we shall call the conditions of localization;

(i) The boundary layer: The problem can be treated along the same line as adopted in the theory of the effective breadth ${ }^{4}$ with respect to the compressive strength of a flat plate and under an idea similar to the conception due to L. Prandtl concerning the motion of a viscous fluid ${ }^{5}$ ). The scale of distribution of the incompatible disturbance $w$ must be so small compared with the size of the test-piece that the distribution of $w$ is to go rapidly to a state of uniformity as we go into the inner part far from the surface. It may be a sufficient explanation of the independence of the yielding condition of the sizes of the test-pieces so $f a r$ as they have geometrically similar forms.

(ii) The periodicity : It is not necessary that 20 takes a constant value in the deep interior. But a subgroup of the group of motions which defines the Euclidean manifold connected with the elastic body before yielding /must hold for the distribution of $v$. We have therefore to consider that the incompatible deformation must be of a periodic distribution outside the boundary layer. An analogous phenomenon may be practically observed in connection with the flat plate in such a state that the theory of the effective breadth holds.

By the above criterions we may further consider that the curvature of the boundary is so small that it may be treated to be flat, the radius of curvature being very large compared with the period of distribution of $w$. Then the $v$-functions satisfying the equation of equilibrium contains sine or cosinefunctions in conformation of the group of motions. Among them we must further select those which satisfies the boundary conditions. The possible period may still be numerous, of an infinite number, promising an infinite variety of the critical values, the smallest of which has usually the longes: wave of $w$. Its half wave length must be nearly the whole length of the test-piece. It is a contradiction to the condition of the boundary layes. What really appear must be of much smallex wavelength.

What is then the criterion to determine the period of $w$ ? The author says that it can be sought among the microscopic properties of the material. We cannot conceive an wave-length which is less than the lower limit of the linear dimension under which the mean elasticity cannot hold. A wavelength much above this limit may happen. Still may it receive certain restrictions from the fundar mental length for it is quite reasonable that any change in the microscopic structure produces. distribution with a period comparable to the fun damental length. We shall therefore propose a constant value $\lambda$ for the wave-length of the distri bution of $w$. It is essentially the same with the quantity $h$ in the previous paper. ") Only such incompatible deformations are permitted that the smallest period of the distribution in all possible directions is equal to $\lambda$ in virtue of isotropy.

The required characteristic value must accordingly be determined by the equations $(1 \cdot 1)$ and $(1 \cdot 2)$ in consideration of the conditions of localization.

\section{\& 2. The Equation of Equilibrium.}

Let the free surface of the test-piece be $z=0$, then one of the principal axes of the stress does necessarily agree with the axis of $z$. Take the directions of the other principal axes as the axes of $x$ and $y$ (Fig. 1), then fundamental equation of equilibrium assumes the form 


$$
B u \Delta w+\sigma_{x} \frac{\partial^{2} w}{\partial x^{2}}+\sigma_{y} \frac{\partial^{2} w}{\partial y^{2}}+\sigma_{z} \frac{\partial^{2} w}{\partial z^{2}}=0
$$

and the boundary conditions require that

$$
(1+\kappa) \frac{\hat{\sigma}^{2} w}{\partial^{2}}-\kappa \Delta v=0
$$

$$
\text { and }
$$

$$
(1+\kappa) \frac{\partial^{3} w}{\partial z^{3}}-(2+\kappa) \frac{\partial \Delta w}{\partial z}-\frac{\sigma_{z}}{B} \frac{\partial w}{\partial z}=0
$$

at every point of the free surface $z=0$.

The solution of $(2 \cdot 1)$ suitable to the conditions discussed in the last section must be of the form

$$
\imath v=W e^{\alpha x+\beta y+\gamma z},
$$

where $W$ is an arbitrary constant, $\alpha$ and $\beta$ are pure inaginary and the real part of $\gamma$ cannot be positive. Substituting $(2: 3)$ into $(2 \cdot 1)$, we get

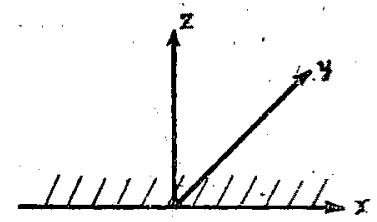

FIg. 1.

$$
\begin{aligned}
& \left(\omega^{2}+\gamma\right)^{2}+\frac{\sigma_{z}}{B}\left(\omega^{2}+\gamma^{2}\right) \\
& +\frac{\sigma_{x}-\sigma_{z}}{B} \alpha^{2}+\frac{\sigma_{y}-\sigma_{z}}{B} \beta^{2}=0
\end{aligned}
$$

where:

$$
\omega^{2}=\alpha^{2}+\beta^{2} \text {. }
$$

The equation( $(2 \cdot 4)$ may be regarded as a quadratic equation with respect to $\omega^{2}+\gamma^{2}$. Solving it, we get

$$
\begin{aligned}
& \omega^{2}+\gamma^{2}=\frac{1}{2}\left[-\frac{\sigma_{z}}{B}\right. \\
& \pm \sqrt{\left.\left(\frac{\sigma_{z}}{B}\right)^{2}-4 \frac{a^{2}\left(\sigma_{x}^{2}-\sigma_{z}\right)+\beta^{2}\left(\sigma_{y}-\sigma_{z}\right)}{B}\right]}
\end{aligned}
$$

vor

$$
r^{2}=\omega^{2}\left( \pm \mu-1-\frac{\nu^{2}}{2}\right)
$$

where

$$
\begin{aligned}
& \mu=\sqrt{\rho^{4}+\frac{\nu^{4}}{4}}, \nu^{2}=\frac{\sigma_{z}}{\omega^{2} B}, \\
& \rho^{4}=-\frac{\alpha^{2}\left(\sigma_{x}-\sigma_{\nu}\right)+\beta^{2}\left(\sigma_{y}-\sigma_{z}\right)}{\omega^{4} B} .
\end{aligned}
$$

The four possible characteristic values of : $r$ may be real or complex according as the values of $\omega^{4}$, $\beta^{4}, \nu^{4}$. Among them we have to put aside such as are inconsistent with the boundary conditions and the condition of localization.

\section{\& 3. Discussion of the Condition of Localization.}

We shall firșt discuss the condition of localization.

(I) When $\omega^{2}=0$, we have $\alpha^{2}=\beta^{2}=0$ and therefore

$$
\eta^{2}=\dot{0},-\frac{\sigma z}{B}
$$

If $-\sigma_{z} / B>0$, it makes the distribution of $v$ aperiodic with respect to every direction. This case must be omitted by virtue of the condition of periodicity. Hence we have always to expect

$$
\frac{\sigma_{z}}{B}>0
$$

only, therefore

$$
\gamma= \pm i \sqrt{-\frac{\sigma_{z}}{B}}
$$

(II) When $\omega^{2} \neq 0, \omega^{2}$ is always negative. In par ticular, (II-1) If $\rho^{4}>1+\nu^{2}$ i.e. $\mu-1-\frac{\nu^{2}}{2}>0$, $-\mu-1-\frac{\nu^{2}}{2}<0$, one of the $\gamma^{2 y} s$ is positive and the other negative. Hence the characteristic values consistent with the condition (i) are only

$$
\left.\begin{array}{l}
\gamma_{1}=-\sqrt{\omega^{2}\left(-\mu-1-\frac{\nu^{2}}{2}\right)}<0 \\
r_{2,3}= \pm \omega \sqrt{\mu-1-\frac{\nu^{2}}{2}} .
\end{array}\right\}
$$

$\gamma_{1}$ is real, negative and $\gamma_{2}, \gamma_{3}$ are pure imaginary.

The possible form of $w$ can be expressed as a linear combination of the three fundamental systems in the form

$$
\left.\begin{array}{rl}
u & =e^{\alpha x+\beta y}\left\{\left(W_{1}+i W_{1}^{\prime}\right) e^{\gamma_{1} z}+\left(W_{2}+i W_{2}\right) e^{\gamma_{2} z}\right\} \\
& +e^{-\alpha x-\beta y}\left\{\left(W_{1}-i W_{1}^{\prime}\right) e^{\gamma} z^{z}+\left(W_{2}-i W_{2}^{\prime}\right) e^{-\gamma_{2} z}\right\}
\end{array}\right\}
$$

consisting of a pair of conjugatè complex functions. Substituting it in the boundary conditions $(1 \cdot 2)$, putting the coefficients of $e^{\alpha x+\beta y}, e^{-\alpha z-\beta y}$ severally equal to zero we obtain four relations. 
Eliminating $W_{1}, W_{1^{\prime}}, W_{\text {, }}$, $W_{2}^{\prime}$ from them, we shall have a real equation at last. But $\gamma_{1}\left[-\gamma_{1}^{2}-(2+\kappa) \omega^{2}-\frac{\sigma_{z}}{B}\right], \quad \gamma_{2}^{2}\left[-\gamma_{2}^{2}-(2+\kappa) \omega^{2}-\frac{\sigma_{z}}{B}\right]=0$ the procedures and the result are quite the same as the elimination of two complex constants $W_{1}+i W_{1}^{\prime}$, and $W_{2}+i W_{2}^{\prime}$ from the equations obtained by consider ing the first line of (3.4), the one of the conju gate complex quantities.

(II-2) If $\rho^{4}=1+\nu^{2}$ i.e. $\mu-1-\frac{\nu^{2}}{2}=0,-\mu-1$ $-\frac{\nu^{2}}{2}<0$, one of the $\gamma^{2}$ 's is zero and the other is positive. Hence $\gamma$ 's permitted are zero and negative in virtue of the localization condition.

$$
\text { (II - 3) If }-\frac{\nu^{4}}{4}>\rho^{4}>1+\nu^{2} \text { i.e. } \pm \mu-1-\frac{\nu^{2}}{2}<0,
$$

For the case (I) especially, we have

$$
r=0
$$

or

$$
\gamma_{1}=-\gamma_{z}, \quad \gamma^{2}-\frac{\sigma_{z}}{B}=0
$$

by virtue of $\omega^{2}=0$. These conditions agree with $(3 \cdot 1)$.

For the case (II), we have

we have two negative values for $r, r^{2}$ being always positive, irrespective of the signs

$$
\begin{gathered}
\omega^{2}\left(\mu-1-\kappa-\frac{\nu_{2}}{2}\right) \\
\omega^{2} \sqrt{\mu-1-\frac{\nu^{2}}{2}}\left(-\mu-1-\kappa-\frac{\nu^{2}}{2}\right)
\end{gathered}
$$

$$
\begin{aligned}
& \omega^{2}\left(-\mu-1-\kappa-\frac{\nu^{2}}{2}\right) \\
& \omega^{2} \sqrt{-\mu-1-\frac{\nu^{2}}{2}}\left(\mu-1-\kappa-\frac{\nu^{2}}{2}\right)=0
\end{aligned}
$$

$(\mathrm{Il}-4)$ When $\rho^{4}+\frac{\nu^{4}}{4}<0, \mu$ being pure imaginary $i . e$. when $\left(\sigma_{x}-\sigma_{z}\right) / B$ ox $\left(\sigma_{y}-\sigma_{z}\right) / B$ takes large negative value, $\gamma^{2}$ takes conjugate complex values. Taking the square roots we obtain $\gamma^{\prime} \mathrm{s}$ with positive as well as negative value of the real part. But the latter only is compatible with the condition at the point of infinity mentioned in (i).

We obtain, therefore, one fundamental system for the distribution in the direction of $z$ from each of the double signs. Fundamental systems of other kinds cannot be permitted throughout the cases (1), and (II).

\section{\& 4. The Boundary Conditions}

We have seen that the dislocation function $w$ must have the form

$$
\text { we } \quad w=e^{\alpha-x+\beta v}\left(W_{1} e^{\gamma_{1} z}+W_{2} e^{\gamma_{2} z^{z}}\right)
$$

in any way. By substifuting (4.1) in the equation $(2 \cdot 2)$, we get

$$
\left.\begin{array}{l}
\left(r_{1}^{2}-\kappa \omega^{2}\right) W_{1}+\left(\gamma_{2}^{2}-\kappa \omega^{2}\right) W_{2}=0 \\
r_{1}\left[-r_{1}^{2}-(2+\kappa) \omega^{2}-\frac{\sigma_{z}}{B}\right] W_{1} \\
-r_{2}\left[-r_{2}-(2+\kappa) \omega^{2}-\frac{\sigma_{z}}{B}\right] W_{2}=0
\end{array}\right\}
$$

where the common factor $e^{\alpha x+\beta y}$ is comitted.

Eliminate $W_{1}$ and $W_{2}$ from(4.2), we get then since each of the double signs in(2.5.1) affords a permissible characteristic value of $r$ consistent with the condition of localization.

Write now

$$
\left.\begin{array}{l}
A=\frac{\mu}{1+\frac{\nu^{2}}{2}} \\
K=\frac{\kappa}{1+\frac{\nu^{2}}{2}},
\end{array}\right\}
$$

then the equation $(4 \cdot 3 \cdot 1)$ becomes

$$
\begin{aligned}
& \left|\begin{array}{ll}
A-1-K & -A-1-K \\
\sqrt{ } \overline{A-1}(-A-1-K) & \sqrt{-\Lambda-1}(A-1-K)
\end{array}\right|=0 \\
& \sqrt{-\Lambda-1}(A-1-K)^{2}=\sqrt{\Lambda-1}(A+1+K)^{2}
\end{aligned}
$$

It may further be led to

$$
A\left[\Lambda^{4}+2(1-K)(3 K-1) A^{2}+(1-K)^{3}(K-3)\right]=0
$$

by squaring each member and rearranging the result.

But $A=0($ i.e. $\mu=0$ ) is meaningless for we have $\gamma_{1}=\gamma_{2}$ in this case so that the two fundamende 
systems coincide to each other. The required equation for the characteristic values is then

$$
\Lambda^{4}+2(1-K)(3 K+1) \Lambda^{2}+(1+K)^{3}(K-3)=0 \text {. }
$$

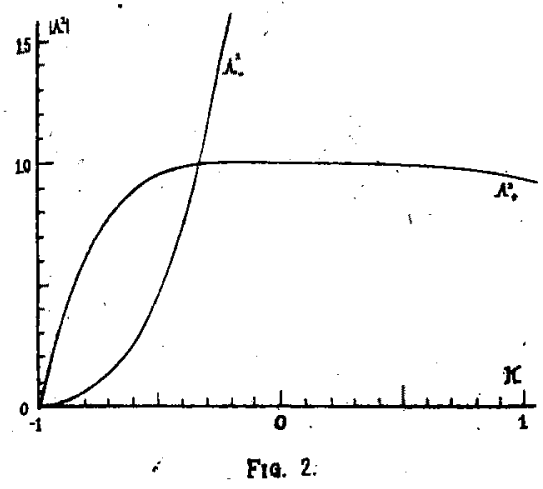

It gives two values of $A^{2}$

$$
\left.\begin{array}{l}
A^{2}=A^{2}{ }_{+}=-(1+3 K)+2 \sqrt{(1+K)^{2}+K^{2}} \\
A^{2}=A^{2}=-(1+3 K)-2 \sqrt{(1+K)^{2}+\bar{K}^{2}}
\end{array}\right\}
$$

Their numerical values are calculated for

$$
K>-1 \text {. }
$$

and plotted in Fig 2.

It is noteworthy that $\Lambda^{2}$ is nearly equal to unity for $K>-0.3$ almost everywhere and takes the maximum value at $K=0$. We can write then almost everywhere

$$
\gamma=-\tilde{\omega} \sqrt{\left(1+\frac{\nu^{2}}{2}\right)\left(1 \pm \mid A_{+}\right)}
$$

where

$$
\widetilde{\omega}=-i \omega .
$$

One of the values of $\gamma$ given by $(4 \cdot 7)$ is almost equal to zero for almost any value of $\kappa>-0.3$ so that it affords a fundamental system whose amplitude decreases inwards of the test-piece only very slowly. The disturbance caused by it must be of a considerable amplitude not only at the free surface but also in the interior of the test-piece. We shall therefore call the disturbances of the type of $A_{+}$ the "intrusive" type. It contains the case $(I I-1)$, (II-2) and (II-3).

The other root $A^{2}$ - is negative and monotone, decreasing for $K>-1$. The two values of $r$ corresponding to it are conjugate complex and may be written in the form

$$
\gamma=-\widetilde{\sigma} \sqrt{\left(1+\frac{\nu^{2}}{2}\right)\left(1+|\Lambda|^{2}\right)} e^{i}-\arctan \mid \Lambda !
$$

It corresponds therefore to the case $(I I-4)$. Since the absolute value $\sqrt{\tilde{\omega}^{2}\left(1+\frac{\nu^{2}}{2}\right)\left(1+|\Lambda|^{2}\right)}$ is considerably large, each of the fundamental system is oscillatory and the amplitude rapidly decreasing inwards the test-piece. The interior of the testpiece may not be much disturbed by such a'kind of yielding. We shall accordingly call this type the "superficial" type.

Any pair of values of $K$ and $A$ specifies the condition of yielding for any given pair of $B$ and $k$. If the negative and positive $B^{\prime}$ 's be of the same absolute value in conformation to our anticipation in a former report, the compressive and tensile yielding stresses should be of the same absolute value. And it is confirmed experimentally as it is observed in bending tèst of the mild steel beam.

\section{\& 5. The Theory of Maximum Shear.}

The third equation of $(2 \cdot 6)$ can be written

$$
\rho^{4}=-\frac{\sigma_{x}}{B} \frac{\alpha^{2}\left(1+-\beta^{2}-\frac{\sigma_{y}}{\alpha^{2}}-\sigma_{x}\right)}{\omega^{4}}+\nu^{2} .
$$

We have however

$$
\rho^{4}=A^{2}+\Lambda^{2} \nu^{2}+\frac{\Lambda^{2}-1}{4} \nu^{4}
$$

by virtue of $(4 \cdot 4)$. Equating the two expressions for $\rho^{4}$, we get

$$
\begin{aligned}
\frac{\sigma_{x}}{B}= & \frac{\tilde{\omega}^{4}}{\tilde{\omega}^{2}-\tilde{\beta}^{2}\left(1-\frac{\sigma_{y}}{\sigma_{x}}\right)} \times \\
& \times\left[A^{2}+\left(\Lambda^{2}-1\right)\left(\nu^{2}+\frac{\nu^{4}}{4}\right)\right],
\end{aligned}
$$

where

We can assume $\left|\sigma_{x}\right| \geqq\left|\sigma_{y}\right|$ or

$$
\alpha=-i \alpha, \quad \bar{\beta}=-i \beta,
$$

$$
1 \geqq\left|\frac{\sigma_{y}}{\sigma_{x}}\right| \text {, }
$$

without loss of generality. We have then

$$
\frac{\dot{\boldsymbol{\omega}}^{4}}{\widetilde{\omega}^{2}-\bar{\beta}^{2}\left(1-\frac{\sigma_{y}}{\sigma_{x}}\right)} \geqslant \frac{\widetilde{\sigma}^{4}}{\widetilde{\sigma}^{2}}={\overline{\alpha^{2}}}^{2}+\overline{\beta^{2}} \geqq{\overline{\alpha^{2}}}^{2}
$$


Hence the least value of $\sigma_{x}|B|$ happens for $\bar{\beta}^{2}=0$,

Since $w$ can take not only real but also actually pure imaginary and $B$ not only positive but negative also, each of the following pair of equations

$$
\begin{array}{r}
B \Delta \Delta w_{(+)}+\sigma_{x} \frac{\partial^{2} v v_{(+)}}{\partial x^{2}}+\sigma_{y} \frac{\partial^{2} w_{(+)}}{\partial y^{2}}+\sigma_{z} \frac{\partial^{2} v_{(+)}}{\partial z^{2}}=0 \\
(5 \cdot 3) \\
-B \Delta \Delta z v_{(-)}+\sigma_{x} \frac{\partial^{2} z v_{(-)}}{\partial x^{2}}+\sigma_{y} \frac{\partial^{2} w w_{(-)}}{\partial y^{2}}+\sigma_{z^{2}} \partial^{2} w_{(-)}=0
\end{array}
$$

must hold independently. There may be a certain mutual constraint between $v v_{(\rightarrow)}$ and $v_{(\rightarrow)}$ owing to the condition of localization or the existence of the fundamental length. The corresponding equations of equilibrium and the boundary conditions occur independently. They might be regarded as the components of displacement in the directions of the fourth and fifth dimensions perpendicular to the original flat manifold.

For the smallest load compatible with the condition $(5 \cdot 2)$, the equation $(5 \cdot 3)$ becomes

$$
B \Delta \Delta w_{(+)}+\sigma \frac{\partial^{2} w_{(+)}}{\partial x^{2}}+\sigma_{z} \frac{\partial^{2} w_{(+)}}{\partial z^{2}}=0
$$

The equation $(5 \cdot 4)$ must be then

$$
-B \Delta \Delta v_{(\rightarrow)}+\sigma_{y} \frac{\partial^{2} w_{(-)}}{\partial y^{2}}+\sigma_{z} \frac{\partial^{2} w w_{(-)}}{\partial z^{2}}=0
$$

because the condition of the least load cannot be satisfied, if it is not the case, $\bar{\alpha}^{2}$ and $\beta^{2}$ appearing in the same equation of equilibrium.

From(5*3.1)we get

$$
-\sigma_{B}=\bar{a}^{2}\left[A^{2}-\left(A^{2}-1\right)\left(\nu^{2}+\frac{\nu^{4}}{4}\right)\right]
$$

and similarly from $(5 \cdot 4 \cdot 1)$

$$
-\frac{\sigma_{y}}{B}=\bar{\beta}^{2}\left[A^{2}+\left(A^{2}-1\right)\left(\nu^{2}+\frac{\nu^{4}}{4}\right)\right] .
$$

Although the equation (5.5) and $(5 \cdot 6)$ are entirely independent, there is a relation such as follows among the frequencies $\bar{a}^{2}$ and $\bar{\beta}^{2}$ in virture of the restriction owing to the fundamental length.

The corresponding wave-length be

$$
\lambda_{x}=\frac{2 \pi}{\bar{a}}, \quad \lambda_{y}=\frac{2 \pi}{\bar{\beta}}
$$

respectively in the directions of $x$ and $y$, for the displacement in the direction of fourth and fifth dimension: Since they are independent distributions we cannot introduce a definite parallelogram of period such as shown in Fig. 3. Else the condition of independence is violated, for there should be introduced a definite functional relation among them:

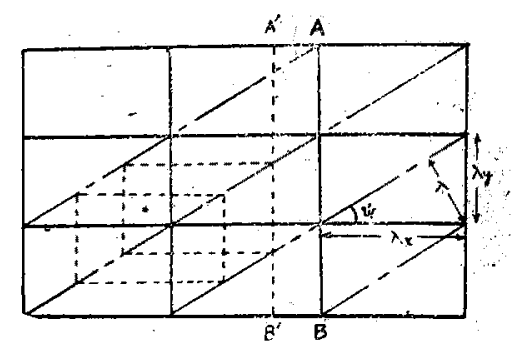

FIE 3

Therefore, a specific longitudinal (or transverse) distribution can appear not only on a specific lon gitudinal (or transverse) line $A B$ for example but on any other longitudinal or (transverse) line $A^{\prime} B^{\prime}$ also. In short the positions of the longitudinal and transverse mesh-lines are indeterminate. Only their directions are determinate. Hence all the point on the diagonal line must be consideref equivalent as well as the vertices and sides of the rectangle. The distance between the adjacent diagonals of the rectangular mesh is given by

$$
\lambda=\lambda_{2} \sin \psi=\lambda_{y} \cos \psi
$$

where the angle between the diagonal and the axis of $x$ is denoted by $\psi$. It is less than each $\lambda_{x}$ and $\lambda y$. Hence it must be equal to the smallest wave length aforementioned. We obtain therefore

$$
\bar{a}^{2}+\overline{\beta^{2}}=\left(\frac{2 \pi}{\lambda}\right)^{2}=\text { const. }
$$

from $(5 \cdot 7), \lambda$ being considered constant. This is nothing else than the mathematical expression of the existence of the fundamental wave length.

Similarly we have for (1)

$$
\bar{a}^{2}+\bar{\gamma}^{2}=\left(\frac{2 \pi}{\lambda}\right)^{2}, \quad \bar{\beta}^{2}+\bar{\gamma}^{2}=\left(\frac{2 \pi}{\lambda}\right)^{2}
$$

where

$$
\bar{\gamma}=-i \gamma
$$


Thus we obtain from $(5.5)$ and $(5 \cdot 7)$

$$
\frac{\sigma_{x}-\sigma_{y}}{B}=\left(\frac{2 \pi}{\lambda}\right)^{2}\left[\Lambda^{2}+\left(\Lambda^{2}-1\right)\left(\nu^{2}-\frac{\nu^{4}}{4}\right)\right]
$$

Similarly from $(3 \cdot 1)$

$$
\begin{aligned}
\frac{\sigma_{x, y}-\sigma_{x} \Lambda^{2}}{B}= & \left(\frac{2 \pi}{\lambda}\right)^{2} \Lambda^{\prime \prime}+\bar{a}^{z}, \bar{\beta}^{2} \\
& \left(\Lambda^{2}-1\right)\left(\nu^{2}+\frac{\nu^{2}}{4}\right)
\end{aligned}
$$

by virtue of $(5 \cdot 8)$ and $(5 \cdot 8 \cdot 1)$

For the intrusive type $A^{2}=A^{2}{ }_{+}=1$, which is to occur before the superficious, the expressions are simplified to

$$
\begin{gathered}
\left|\frac{\sigma_{x}-\sigma_{y}}{B}\right|=\left(\frac{2 \pi}{\lambda}\right)^{2}, \quad\left|\frac{\sigma_{x}-\sigma_{z}}{B}\right|=\left|\frac{2 \pi}{\lambda}\right|^{2}, 1 \\
\left|\frac{\sigma_{y}-\sigma_{z}}{B}\right|=\left|\frac{2 \pi}{\lambda}\right|^{2} .
\end{gathered}
$$

What is the smallest among these values will naturally take place at first. The conclusion is entirely coincident with the theory of maximum shear which has been considered as well established by a great number of experimental facts.

\section{Conclusion.}

Since we have deduced, starting from the three principal propositions in the introduction, results coincident with the theory of maximum shear at least for the uniform stress, correctness of our theory may be considered well proved. Although there may not be any objection concerning the introduction of the boundary layer (i), we may expect some disputes about the existence. of the fundamental length and its expressions (5.11).

That the contraint due to the equation of equiliw brium is so predominant as to afford a regular periodicity even to such material as with a nonuniform microscopic structure can, however be offered as a sufficient argument. And what is more, it requires anly periodicity but interferes not with the wavelength.
What appears most remarkáble compared with the common theory of buckling, might be the separation of the equations $(5 \cdot 3 \cdot 1)$ and $(5 \cdot 4 \cdot 1)$ followed by the reconnection by $(5 \cdot 8)$ and $(5 \cdot 8 \cdot 1)$. But it should be the most essential in relation to the yielding phenomena, based on the irreversibility, the laws of thermodynamics and the condition of the smallest load.

We have been informed about such experimental facts that cannot be explained by a simple theory of the maximum shear6),7). Some representatives of such cases will be treated analytically in the forthcoming reports II and III.

\section{Bibliography}

1). K. Kondo : A Proposal of a New Theory concerning the Yielding: of Materials based on Riemannian Geometry I-1I. Journ. Japan Soc. Appl. Mech., Vol. 2, No. 11 (1949) pp. 123/128; Vo1. 2, No. 12(1949) pp. 146/151.

2). K. Kondo: On the Dislocation, the Group of Holonomy and the Theory of YieIding. ibid. Vol. 3, No. 17(1950). pp. $11 / 14$.

3). K. Kondo: On the Fundamental Equations of the Theory of Yielding. ibid. vol. 3 No. 20 (1950) pp 184/188.

4). Th. VON KARMAN,E.E.SCHLER,L.H.DONNEL: Strength of Thin plates in Compression. Applied Mechanics, A.S.M.E. (1932)pp. 54/55.

5). L. PrandTL: Über die Flüssigkeitsbewegung mit sehr kleinen Reibung. Vier Abhandiungen, zur Hydrodynamik und Aerodynamik, Göttingen, (1927).

6). F. NAKANISHI: On the Yield Point of Mild Steél. Aeronautical Res. Inst. T.I.U., Report. Vol. 6, No. $72(1931)$ pp. 83/142. .

7). F. NaKanishn: On the Yield Points of Mild Steel Beams under Uniform Bending. ibid. Vol. 8, No. 104 (1934) pp. 271/289. 